\title{
Seguimiento del gesto de señalar y de la mirada en estadios tempranos del desarrollo
}

\author{
Follow- up the gesture of pointing and gazing at early stages \\ of development
}

\begin{abstract}
Angel M. Elgier ${ }^{1,2,3,4,5^{*}}$, Lucas G. Gago Galvagno ${ }^{1,2,3,4,5}$, Gonzalo Clerici ${ }^{5}$, Camila Tortello ${ }^{5}$ \& Susana C. Azzollini ${ }^{3,4,5,6,7}$ ${ }_{1}^{1}$ Universidad de Buenos Aires, ${ }^{2}$ Facultad de Psicología y Relaciones Humanas de la Universidad Abierta Interamericana, ${ }^{3}$ CONICET, ${ }^{4}$ Instituto de Investigaciones en Psicología, ${ }^{5}$ Facultad de Psicología de la Universidad de Buenos Aires, ${ }^{6}$ Centro de Investigaciones Sociales y Humanas para la Defensa (CISOHDEF), ${ }^{7}$ Secretaria de Ciencia y Técnica de la Facultad del Ejército de la Universidad de la Defensa Nacional (UNDEF)
\end{abstract}

\section{RESUMEN}

La atención conjunta, definida como la coordinación visual de dos individuos dirigida hacia un objeto de interés mutuo, es una habilidad nuclear que se desarrolla durante la infancia temprana y es la base de la futura capacidad lingüística, emocional, de la teoria de la mente y de la cognición en general de toda persona. Este fenómeno incluye la adquisición de comportamientos como el seguimiento de mirada y señalamiento. Por otro lado, la vulnerabilidad social es un factor de riesgo estudiado en relación con numerosas variables como la memoria, las funciones ejecutivas y distintas patologías. En este caso, se propone investigar la modulación que ejerce la vulnerabilidad social sobre las habilidades de seguimiento de mirada y señalamiento en la primera infancia. La muestra estuvo conformada por 18 infantes entre 9 a 13 meses. Se midieron las conductas de comunicación a partir del seguimiento de la mirada y del señalamiento del infante a objetos distantes señalizados por el evaluador. Se midió la latencia y duración de la conducta de seguimiento a través del análisis conductual aplicado. La vulneravilidad social fue medida utilizando la Escala de Nivel Económico Social (NES). Los resultados reflejan que no se encuentran diferencias en las capacidades de seguimiento de la mirada y señalamiento según el nivel socioeconómico. Estos resultados resaltan que a pesar de que el nivel socioeconómico produce limitaciones en las habilidades comunicativas, no habría aún efectos visibles en los primeros estadios del desarrollo.

Palabras clave: Comunicación, desarrollo, vulnerabilidad social, infancia, atención conjunta.

\begin{abstract}
Joint attention, defined as the visual co-ordination of two individuals directed towards an object of mutual interest, is a nuclear skill that develops during early childhood and is the basis of the future linguistic, emotional capacity of mind theory and Of the general cognition of every person. This phenomenon includes the acquisition of behaviors such as the tracking of gaze and signaling. On the other hand, social vulnerability is a risk factor studied in relation to many variables such as memory, executive functions and different pathologies. In this case, it is proposed to investigate the modulation that social vulnerability exerts on the abilities of followup of gaze and signaling in early childhood. The sample consisted of 18 infants between 9 and 13 months. Communication behaviors were measured from the follow-up of the gaze and from the infant's pointing to distant objects marked by the evaluator. The latency and duration of follow-up behavior were measured through applied behavioral analysis. Social vulnerability was measured using the Social Economic Level Scale (NES) (INDEC, 2000). The results reflect that there are no differences in the abilities to follow the look and pointing according to the socioeconomic level. These results highlight that although the socioeconomic level produces limitations in communicative skills, there would still be no visible effects in the early stages of development. Further research must be carried out to corroborate this claim.
\end{abstract}

Keywords: Communication, development, social vulnerability, infancy, joint attention.

Historial del artículo:

Recibido, 18 de octubre de 2016; aceptado, 1 de febrero de 2017; disponible en línea, 25 de junio de 2017

* Investigador de la Facultad de Psicología de la Universidad de Buenos Aires - CONICET; y, Facultad de Psicología y Relaciones Humanas de la Universidad Abierta Interamericana.

Correo: amelgier@gmail.com

Este es un artículo de acceso abierto, licencia CC BY (https://creativecommons.org/licenses/by/4.0/) 


\section{INTRODUCCIÓN}

Al nacer, el infante cuenta con competencias comunicativas de tipo no verbal, llamadas protolingüísticas porque son la base en las que se asentaran en un segundo momento las capacidades verbales y compolejas propas del lenguaje. Dentro de estas competencias de comunicación incipientes encontramos las habilidades de atención conjunta, que se definen como la capacidad de los infantes de mirar en forma alternada entre un objeto y un adulto, coordinando la mirada en pos de dirigir la atención hacia un objetivo de interés mutuo. Estas interacciones entre un niño, un adulto y un objeto son esenciales para el desarrollo del conocimiento en general, y específicamente para el conocimiento social (Carpendale \& Lewis, 2004). En tal sentido, es un componente esencial de la cognición social, la comunicación, la teoría de la mente y la adquisición del lenguaje (Bruner, 1995; Tomasello \& Carpenter, 2005).

La atención conjunta posee diversidad de definiciones, pero todas ellas apuntan a una interacción recíproca y simultanea entre dos o más individuos, centrándose mentalmente en uno mismo y en el mismo objeto externo (Baldwin, 1995). En su definición acotada, refiere simplemente a mirar hacia donde alguien está mirando, y en su definición amplia inculye el inicio, la respuesta a la atención conjunta y el monitoreo de la atención del otro hacia una entidad exterior, con la suposición de que otro está mirando y experimentando algo en el mundo visual (Sigman \& Kasari, 1995; Bruner, 1995; Tomasello, 1995).

Hay dos categorías principales de las conductas de atención conjunta en la infancia. Una es la "respuesta a la atención conjunta" (por sus siglas en inglés, Responding to Joint Attention - RJA), que se refiere a las medidas de capacidad de los bebés para seguir la dirección de la mirada y los gestos de los demás con el fin de compartir un punto de referencia común. Alternativamente, se encuentra la categoría "iniciando la atención conjunta" (por sus siglas en inglés, Initiation of Joint Attention - IJT) que implica generar gestos y contacto visual por parte de los infantes para dirigir la atención de los otros hacia objetos, hacia eventos y hacia ellos mismos. La función prototípica del IJT es "mostrar" o de forma espontánea tratar de compartir intereses o experiencia placentera con otros (Mundy \& Jarrold, 2010).

Entre las habilidades de atención conjunta en infantes encontramos las referidas al seguimiento de la mirada y el señalamiento, y a la producción de gestos para llamar la atención de los adultos. No sólo contribuyen al desarrollo intelectual, al desarrollo socio-emocional y al desarrollo del lenguaje (e.g. Carpenter, Nagell, \& Tomasello, 2005; Sheinkopf, Mundy, Claussen, \&
Willoughby, 2004; Ulvund \& Smith, 1996) si no que además el desarrollo anómalo de las mismas son indicadores de posibles trastornos del espectro autista o atencionales (Murza \& Schwartz, 2016; BottemaBeutelm, 2016).

En esta línea, se encontraron asociaciones entre la respuesta a la atención conjunta a los 11 meses y comprensión del lenguaje a los 14 y 18 meses, pero no asi en la producción (Brooks y Meltzoff, 2005). La interpetación que realizaron es que los niños que realizan la conexión entre la atención del adulto y los objetos puede generar mejores conexiones entre la etiqueta verbal y el objeto, al compartir los sonidos del habla propios de esta edad. Esto llevaría a un esfuerzo protodeclarativo por parte del niño (Brooks y Meltzoff, 2005). También Morales y colaboradores $(2000)$ encontraron que las diferencias individuales a los 6, 8, 10 y 18 meses estaban relacionadas significativamente con el lenguaje (outcome) a los 30 meses, a partir de la correlación entre el seguimiento del señalamiento y un reporte realizado por los padres de los infantes.

Trevarthen (1990) denominó intersubjetividad primaria a la capacidad innata que tienen los infantes de compartir un significado con otro. En un primer momento lo que se comparte es emocional y automático, el bebé responde a las expresiones afectivas de los adultos. Cuando lo hace, en cierta medida las reconoce y, además, las diferencia de las respuestas que da a otros estímulos no sociales. Es aquí donde se ubicarían el seguimiento de la mirada y el señalamiento. Ya con la aparición de la "iniciación de la atención conjunta", el infante expresa una verdadera intención comunicativa y una capacidad de comprender a los otros como entidades diferentes de su "yo". Según Thevarten, es en ese momento cuando el infante manifiesta una intersubjetividad secundaria, es decir, una motivación deliberada por compartir los intereses y las experiencias con otras personas (Escudero, Carranza \& Huescar, 2013).

Por otro lado, el desarrollo cognitivo infantil puede ser modulado por varios factores individuales (e.g., temperamento, genética) y ambientales (e.g., condición socioeconómica, tipo de crianza, salud mental materna). El nivel socioeconómico por ejemplo modula el desempeño en procesos de control cognitivo (Farah \& Noble, 2004; Lipina, Martelli, Vuelta \& Colombo, 2005). Durante el primer año de vida, la pertenencia a hogares con Necesidades Básicas Insatisfechas (NBI), se asoció a niveles de desempeño más bajos en tareas con demandas de procesos de control cognitivo (i.e., control inhibitorio, memoria de trabajo) (Lipina, Martelli, Vuelta \& Colombo, 2005). A su vez, se han podido identificar asociaciones causales específicas entre (1) los niveles de estimulación ambiental en el hogar y el desempeño en pruebas de lenguaje; y (2) las pautas de crianza parental y el desempeño en 
tareas con demandas de memoria de trabajo (Farah \& Noble, 2004).

Numerosas investiaciones describen las relaciones entre vivir en entornos de bajo nivel socioeconómico (en adelante ESE) y las consecuencias que traen aparejados en el desarrollo comunicativo de infantes. Schady (2011) buscó estimar la asociación entre la educación de los padres, el vocabulario materno y el desarrollo cognitivo temprano, en una muestra de niños vulnerables en el Ecuador rural. Fueron utilizados los datos de 2118 niños. La escolaridad y el vocabulario de las madres fueron fuertes predictores del desarrollo cognitivo de los niños. La riqueza de los hogares, la altura del niño, el peso y los niveles de hemoglobina explicó sólo una pequeña fracción de las asociaciones observadas. Los niveles de vocabulario de las madres y los niños estaban más fuertemente correlacionados entre los niños mayores en la muestra, lo que sugiere que los efectos de un vocabulario materno más rico serían acumulativos.

Saxon y Reilly (1999) realizan un estudio pionero en donde se pone en relieve los efectos del ESE sobre las habilidades preverbales. Más específicamente, analiza la relación entre el ESE de diferentes díadas madrehijo y su atención conjunta, es decir, su habilidad para concentrarse al mismo tiempo en un mismo objeto o evento externo. Si bien este trabajo no muestra correlaciones significativas entre el ESE y la atención conjunta entre madres y bebés, la importancia de replicar este tipo de investigaciones para ver si se encuentra algún tipo de relación entre estas variables reside en el hecho en que se ha reportado que estas habilidades son precursoras de otros procesos indispensables en el desarrollo del niño, como la teoría de la mente (Kristen, Sodian, Thoermer, \& Perst, 2011) y el lenguaje (Charman, 2003), como ya se ha dicho.

También podríamos pensar que estas funciones son tan básicas que, en consecuencia, no se encuentran diferencias significativas según los contextos de crianza. Rivière (1999) propone una cartografía mental, y ubica a estas capacidades de seguimiento de la mirada y señalamiento como funciones de tipo tres, que se generan en todos los contextos de crianza a través del otro dentro de la cultura. Los contextos de vulnerabilidad social, por más de que traen aparejados dificultades para el desarrollo del infante, parecerían no impactar en estas habilidades básicas de comunicación, ya que en estos entornos la interacción del infante con los adultos tambíen se lleva a cabo.

Sin embargo, McGillion y colaboradores (2016) apuntan a que la complejidad sintáctica y léxica utilizada por los niños en su vocabulario están relacionadas con los niveles educativos de los padres, que se asocian al nivel socioeconómico de la los mismos. La educación de los padres correlaciona de forma positiva con la cantidad de habla directa del niño y la calidad de la respuesta de los padres a la atención de sus hijos, sus gestos y sus vocalizaciones (McGillion, Herbert, Pine, Keren-Portnoy, Vihman, \& Matthews, 2013). Este estudio muestra además que el balbuceo y la educación materna son predoctores de la producción de palabras del niño, mientras que la capacidad de señalamiento predice la habilidad de comprensión de palabras. Sólo a partir de los 18 meses se encontraron relaciones entre la capacidad de señalamiento del infante y el desarrollo del lenguaje. Parecería que la asociación entre señalamiento y desarrollo del lenguaje se incrementa con la edad (Colonnesi, Stams, Koster, \& Noom, 2010).

Otros estudios realizados por Tomasello y sus colaboradores (Tomasello \& Todd, 1983; Tomasello \& Farrar, 1986), examinaron la relación entre el vocabulario del niño y la habilidad de la madre para regular los estados atencionales de su hijo. Encontraron que los padres que actuaban cambiando la interacción iniciada por el niño, tenían hijos con vocabularios más acotados en cuanto a la cantidad de palabras utilizadas que aquellos que se adaptaban a los intereses de su hijo. Cuando las madres organizaban la interacción dirigiendo la atención del niño, en vez de seguirla, sus hijos aprendían menos nombres de objetos y más palabras personales-sociales (Escudero et al., 2013).

Rossin y Buzzella (2011) realizaron una intervención de educación temprana en infantes y niños pertenecientes a contextos vulnerables de la Villa 1-11-14 ubicada en la Ciudad de Buenos Aires. La misma consistía en realizar actividades lúdicas, leer cuentos, generar entornos de interacción, entre otros. Luego de un año y medio de intervención, se observó un aumento del repertorio de palabras, mejora en aspectos expresivos y comprensivos del lenguaje; $y$, en cuanto a las habilidades sociales se logró que los niños respetaran el turno, escucharan al compañero, cooperaran con el otro, percibieran las emociones propias y ajenas y aplicaran normas básicas de convivencia. Se puede apreciar así la importancia de la intervención temprana y las dificultades que tren aparejadas en el desarrollo cognitivo de los niños los contextos vulnerables.

En la misma línea, Stevenson, Roach, Leavitt, Miller y Chapman (1998) examinaron habilidades lingüísticas tempranas de 38 bebés de 8 meses (19 nacidos en forma prematura y 19 nacidos a término) de hogares de clase media. Se hicieron análisis de regresión para evaluar la influencia relativa de las habilidades cognitivas infantiles, la sociabilidad infantil y el estado al nacer como posibles predictores del lenguaje receptivo y el comportamiento infantil vocal. Las habilidades de recepción y producción de lenguaje se asociaron con un mayor rendimiento cognitivo y mayor sociabilidad (Stevenson et al., 1988) 
Se ha visto que los niños provenientes de medios socio-económicos desfavorecidos que evidencian problemas de lectura durante los primeros años de la escuela primaria tienden a presentar problemas lingüísticos durante los siguientes años de su escolaridad parecidos, en ciertos aspectos, a los niños disléxicos (Billard, Bricout, Ducot, Richard, Ziegler, \& Fluss, 2010).

El carácter persistente de estas dificultades ha alertado sobre la necesidad de implementar programas que busquen incentivar el desarrollo del lenguaje desde temprana edad con el fin de evitar que los niños provenientes de medios sociales precarios se vuelvan aún más vulnerables al fracaso escolar más tarde. De hecho, se ha encontrado que los niños preescolares de medios vulnerables con deficiencias lingüísticas pueden mostrar mejoras importantes en sus capacidades de comprensión y de expresión a partir de intervenciones que promuevan las habilidades relacionadas con el lenguaje desde edades tempranas (Dockrell, Stuart, \& King, 2010).

A partir de todo lo recabado se concluye que tanto el nivel socioeconómico como el estilo de crianza, el tipo de interacciones en las díadas madre- hijo y el nivel educativo de los padres entre otros modulan las capacidades comunicativas de los niños durante su desarrollo. A su vez, las capacidades de seguimiento de la mirada y el señalamiento serían predictores de las capacidades lingüísticas más complejas, por eso se hace necesario su estudio. Sin embargo, todavía son necesarias investigaciones que trabajen en los primeros años de vida, para asi generar intervenciones específicas en el área de la protocomunicación y comprender cuáles son las variables que influyen este tipo de comunicación temprana.

Para esto, el objetivo general de este trabajo es analizar la modulación que ejerce la vulnerabilidad social sobre las habilidades de seguimiento de mirada y señalamiento en la primera infancia.

Los objetivos específicos incluyen: (1) Determinar los perfiles de desempeño en tareas con demandas comunicativas a partir de evaluaciones de seguimiento de mirada y de seguimiento del señalamiento; en niños de 9 a 13 meses sin historia de trastornos del desarrollo, provenientes de hogares con y $\sin$ Necesidades Básicas Insatisfechas (NBI, criterio de pobreza). (2) Analizar la modulación de tales desempeños en función de factores socioeconómicos del hogar. Se espera encontrar diferencias en las distintas tareas con demandas comunicativas, moduladas por características socioeconómicas (los niños de hogares con NBI tenderán a expresar desempeños menos maduros).

\section{MATERIAL Y MÉTODOS}

\section{Diseño}

Para cumplir con los objetivos de la investigación se planteó un diseño cuasi-experimental, intersujeto (intergrupos) y transversal. Para analizar los resultados, fueron comparados ambos grupos (Nivel Básico Satisfecho y Nivel Básico insatisfecho) en las capacidades comunicativas tempranas (latencia y duración, que serán resultado de los promedios en cada ensayo).

\section{Sujetos}

La muestra estuvo conformada por 18 infantes $(N=18)$ entre 9 a 13 meses (lengua materna: español; apareados por género), sin historia de trastornos del desarrollo, asistentes a tres jardines maternales públicos de distritos escolares de la Ciudad Autónoma de Buenos Aires. La conformación de la muestra se realizó en función de la condición socioeconómica.

La selección de la muestra sigue criterios estrictos: español como primera lengua materna, la visión y audición normales, sin constancia de enfermedades graves, sin antecedentes familiares de enfermedad psiquiátrica, sin antecedentes de lesiones significativas en la cabeza, convulsiones, o enfermedad neurológica, sin abuso de sustancias materna o dependencia.

Los bebés no deben mostrar síntomas de enfermedad aguda, y debieron nacer a término y con peso y altura adecuada para la edad gestacional. Para ello se realizaron preguntas sobre los historiales clínicos tanto de la madre como del niño. Cada madre antes de la administración de la tarea debió firmar por escrito el consentimiento informado.

Es de destacar que los procedimientos de evaluación presentados siguen los principios recomendados por la American Psychological Association (1992) en el trabajo con niños, establecidos por la Convención Internacional sobre los Derechos del Niño, y lo establecido en la Ley № 26.061 de Protección Integral de los Niñas, Niños y Adolescentes de la República Argentina (lo cual implica consentimiento informado de todos los procedimientos).

\section{Procedimiento y controles}

Para las pruebas se utilizó siempre la misma sala, y el evaluador fue siempre el mismo. Los bebés se sentaron en el regazo de sus madres y mirando al experimentador Se les dijo a las madres que no brinden ningún tipo de ayuda.

Se administraron dos pruebas, y en ambas se 
colocaron cuatro juguetes en diferentes lugares del consultorio. Luego se administraron dos ensayos por prueba. Las tareas descritas a continuación para evaluar capacidades comunicativas se basan en procedimientos desarrollados por Carpenter et al. (1998).

Prueba 1. Seguimiento de la mirada. El niño estará sentado frente a El, quien le dará un juguete. Cuando el niño dirija su atención al juguete, El lo llamará por su nombre, esperará a realizar contacto visual y luego emitirá una vocalización -con gesto facial llamativogirando la cabeza hacia el juguete. El alternará su mirada entre los ojos del niño y el juguete varias veces, y girando la cabeza cada vez.

Prueba 2. Seguimiento del señalamiento. El procedimiento será similar al anterior, pero en este caso El señalará al juguete con su mano derecha, al mismo tiempo que alternará su mirada entre el objetivo y los ojos del niño. Para ambas pruebas, E1 mirará y señalará el juguete hasta que el niño lo ubique o, si no deja de mirar a E1, hasta que E2, que se encontrará observando el rostro del niño, determine que el niño ha visto claramente varios giros de cabeza o señalamientos. Se contabilizará un ensayo como correcto si el niño observa el objeto que El está señalando, dirigiendo su mirada al mismo.

Primero se observó si los infantes fueron capaces de realizar la conducta, y más tarde fueron contabilizadas en segundos, con cronómetros, teniendo en cuenta el tiempo de duración que el niño mantiene fijada su mirada en el objeto, y la latencia desde que el experimentador realiza la conducta hasta que el niño mira el objeto. Sólo se registrará la primera latencia, luego se registrará la duración. Para evitar el efecto de orden las tareas fueron presentadas de forma variada.

Por otro lado, a cada madre se le administró la Escala de Nivel Económico Social (NES) (INDEC, 2000) para estimar el nivel socioeconómico familiar y la presencia de indicadores NBI en el hogar. El estatus o nivel socioeconómico (NSE) es una medida total que combina la parte económica y sociológica de la preparación laboral de una persona y de la posición económica y social individual o familiar en relación a otras personas. Además es un indicador importante en todo estudio demográfico; no sólo tiene en cuenta el ingreso económico de la familia, si no también su nivel educativo, tipo de vivienda, acceso a planes sociales, entre otros, ya que la vulnerabilidad social es un fenómeno multidimensional.

Estudios actuales en América Latina muestran la gran relación de este indicador con el estado de salud, nutrición, el rendimiento estudiantil y la esperanza de vida (UNICEF, 2005). La vulnerabilidad social es una variable multidimensional que tiene en cuenta simultáneamente el tipo de hogar, hacinamiento, acceso a servicios públicos, educación y capacidad económica (Lipina et al., 2005), las cuales son medidas mediante esta escala.

\section{RESULTADOS}

\section{Análisis de datos}

Se realizó la prueba de Shapiro- Wilk para contrastar la normalidad de los datos.

Como los tiempos de latencia y duración no tienen una distribución normal, se utilizó la prueba no paramétrica U de Mann- Whitney para muestras independientes.

Se encontró que ambos grupos (NBI y NBS) realizan la conducta de seguir el gesto de señalar y de seguir la mirada.

Por otra parte, el análisis indica que no hay diferencias significativas $(p>, 05)$ en función del nivel socioeconómico tanto en las medidas de latencia como en las de duración para las conductas de seguir el señalamiento y la mirada.

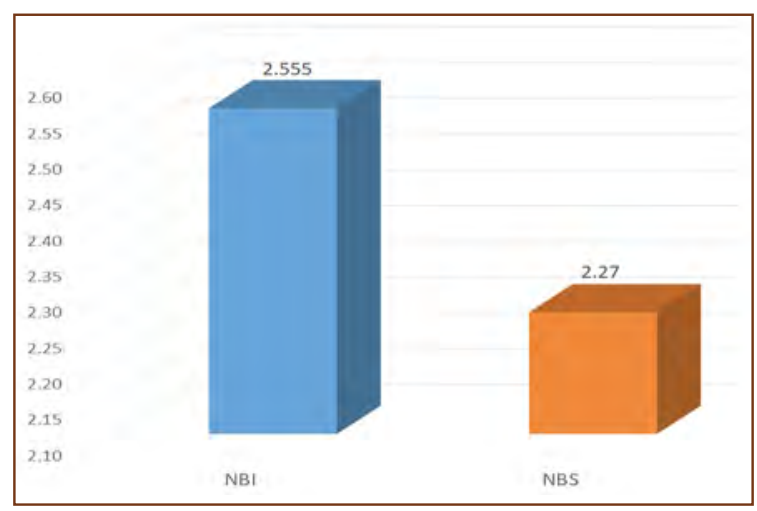

Figura 1.Mediana de la duración en seguimiento del señalamiento para los grupos NBI y NBS.

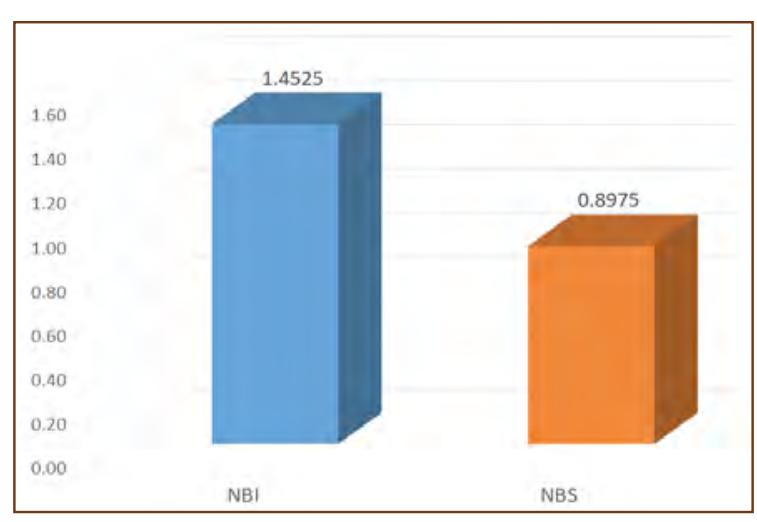

Figura 2. Mediana de la duración en seguimiento de la mirada para los grupos NBI y NBS. 
Tabla 1. Estadísticos descriptivos de la duración del señalamiento y la mirada según el nivel socioeconómico.

\begin{tabular}{|c|c|c|c|c|}
\hline \multicolumn{2}{|c|}{$\begin{array}{c}\text { Grupo } \\
\text { Socioeconómico }\end{array}$} & \multirow[t]{2}{*}{ Estadística } & \multirow{2}{*}{$\begin{array}{l}\text { Resultados } \\
2,590630\end{array}$} & \multirow{2}{*}{$\begin{array}{c}\text { Error típ. } \\
0,525381\end{array}$} \\
\hline Duración del & NBI & & & \\
\hline \multirow[t]{25}{*}{ señalamiento } & & Intervalo de confianza Límite inferior & 1,348300 & \\
\hline & & para la media al $95 \%$ Límite superior & 3,832950 & \\
\hline & & Media recortada al $5 \%$ & 2,534030 & \\
\hline & & Mediana & 2,555000 & \\
\hline & & Varianza & 2,208000 & \\
\hline & & Desv. típ. & 1,486002 & \\
\hline & & Mínimo & 0,625000 & \\
\hline & & Máximo & 5,575000 & \\
\hline & & Rango & 4,950000 & \\
\hline & & Amplitud intercuartil & 1,704000 & \\
\hline & & Asimetría & 0,998000 & 0,752000 \\
\hline & & Curtosis & 1,919000 & 1,481000 \\
\hline & NBS & Media & 2,531880 & 0,321128 \\
\hline & & Intervalo de confianza Límite inferior & 1,772530 & \\
\hline & & para la media al $95 \%$ Límite superior & 3,291220 & \\
\hline & & Media recortada al $5 \%$ & 2,527920 & \\
\hline & & Mediana & 2,270000 & \\
\hline & & Varianza & 0,825000 & \\
\hline & & Desv. típ. & 0,908287 & \\
\hline & & Mínimo & 1,175000 & \\
\hline & & Máximo & 3,960000 & \\
\hline & & Rango & 2,785000 & \\
\hline & & Amplitud intercuartil & 1,325000 & \\
\hline & & Asimetría & 0,237000 & 0,752000 \\
\hline & & Curtosis & $-0,650000$ & 1,481000 \\
\hline Duración de & NBI & Media & 1,888800 & 0,84771 \\
\hline \multirow[t]{25}{*}{ la mirada } & & Intervalo de confianza Límite inferior & $-0,115800$ & \\
\hline & & para la media al $95 \%$ Límite superior & 3,893300 & \\
\hline & & Media recortada al $5 \%$ & 1,686100 & \\
\hline & & Mediana & 1,452500 & \\
\hline & & Varianza & 5,749000 & \\
\hline & & Desv. típ. & 2,397690 & \\
\hline & & Mínimo & 0,000000 & \\
\hline & & Máximo & 7,430000 & \\
\hline & & Rango & 7,430000 & \\
\hline & & Amplitud intercuartil & 2,140000 & \\
\hline & & Asimetría & 2,128000 & 0,75200 \\
\hline & & Curtosis & 5,142000 & 1,48100 \\
\hline & NBS & Media & 1,12560 & 0,38835 \\
\hline & & Intervalo de confianza Límite inferior & 0,20730 & \\
\hline & & para la media al $95 \%$ Límite superior & 2,04390 & \\
\hline & & Media recortada al $5 \%$ & 1,05850 & \\
\hline & & Mediana & 0,89750 & \\
\hline & & Varianza & 1,20700 & \\
\hline & & Desv. típ. & 1,09842 & \\
\hline & & Mínimo & 0,00000 & \\
\hline & & Máximo & 3,46000 & \\
\hline & & Rango & 3,46000 & \\
\hline & & Amplitud intercuartil & 1,23000 & \\
\hline & & Asimetría & 1,48600 & 0,75200 \\
\hline & & Curtosis & 2,68200 & 1,48100 \\
\hline
\end{tabular}


Tabla 2. Estadísticos descriptivos de la latencia del señalamiento y la mirada según el nivel socioeconómico.

\begin{tabular}{|c|c|c|c|c|}
\hline \multicolumn{2}{|c|}{$\begin{array}{c}\text { Grupo } \\
\text { Socioeconómico }\end{array}$} & \multirow[t]{2}{*}{ Estadística } & \multirow{2}{*}{$\begin{array}{r}\text { Resultados } \\
2,97375\end{array}$} & \multirow{2}{*}{$\begin{array}{r}\text { Error típ. } \\
0,85387\end{array}$} \\
\hline Latencia del & $\mathrm{NBI}$ & & & \\
\hline \multirow[t]{25}{*}{ señalamiento } & & Intervalo de confianza Límite inferior & 0,95467 & \\
\hline & & para la media al 95\% Límite superior & 4,99283 & \\
\hline & & Media recortada al $5 \%$ & 2,83583 & \\
\hline & & Mediana & 2,24250 & \\
\hline & & Varianza & 5,83300 & \\
\hline & & Desv. típ. & 2,41511 & \\
\hline & & Mínimo & 0,78500 & \\
\hline & & Máximo & 7,64500 & \\
\hline & & Rango & 6,86000 & \\
\hline & & Amplitud intercuartil & 3,53600 & \\
\hline & & Asimetría & 1,06900 & 0,75200 \\
\hline & & Curtosis & 0,57800 & 1,48100 \\
\hline & \multirow[t]{13}{*}{ NBS } & Media & 1,459380 & 0,389161 \\
\hline & & Intervalo de confianza Límite inferior & 0,539160 & \\
\hline & & para la media al 95\% Límite superior & 2,379590 & \\
\hline & & Media recortada al $5 \%$ & 1,353470 & \\
\hline & & Mediana & 0,922500 & \\
\hline & & Varianza & 1,212000 & \\
\hline & & Desv. típ. & 1,100712 & \\
\hline & & Mínimo & 0,830000 & \\
\hline & & Máximo & 3,995000 & \\
\hline & & Rango & 3,165000 & \\
\hline & & Amplitud intercuartil & 0,979000 & \\
\hline & & Asimetría & 2,212000 & 0,752000 \\
\hline & & Curtosis & 4,916000 & 1,481000 \\
\hline Latencia de & $\mathrm{NBI}$ & Media & 6,390630 & 1,175502 \\
\hline \multirow[t]{25}{*}{ la mirada } & & Intervalo de confianza Límite inferior & 3,611000 & \\
\hline & & para la media al 95\% Límite superior & 9,170250 & \\
\hline & & Media recortada al $5 \%$ & 6,466250 & \\
\hline & & Mediana & 6,485000 & \\
\hline & & Varianza & 11,054000 & \\
\hline & & Desv. típ. & 3,324823 & \\
\hline & & Mínimo & 1,420000 & \\
\hline & & Máximo & 10,000000 & \\
\hline & & Rango & 8,580000 & \\
\hline & & Amplitud intercuartil & 6,731000 & \\
\hline & & Asimetría & $-0,455000$ & 0,752000 \\
\hline & & Curtosis & $-1,198000$ & 1,481000 \\
\hline & NBS & Media & 5,928130 & 0,867683 \\
\hline & & Intervalo de confianza Límite inferior & 3,876380 & \\
\hline & & para la media al 95\% Límite superior & 7,979870 & \\
\hline & & Media recortada al $5 \%$ & 5,900140 & \\
\hline & & Mediana & 6,090000 & \\
\hline & & Varianza & 6,023000 & \\
\hline & & Desv. típ. & 2,454177 & \\
\hline & & Mínimo & 2,360000 & \\
\hline & & Máximo & 10,000000 & \\
\hline & & Rango & 7,640000 & \\
\hline & & Amplitud intercuartil & 3,591000 & \\
\hline & & Asimetría & $-0,076000$ & 0,752000 \\
\hline & & Curtosis & 0,203000 & 1,481000 \\
\hline
\end{tabular}




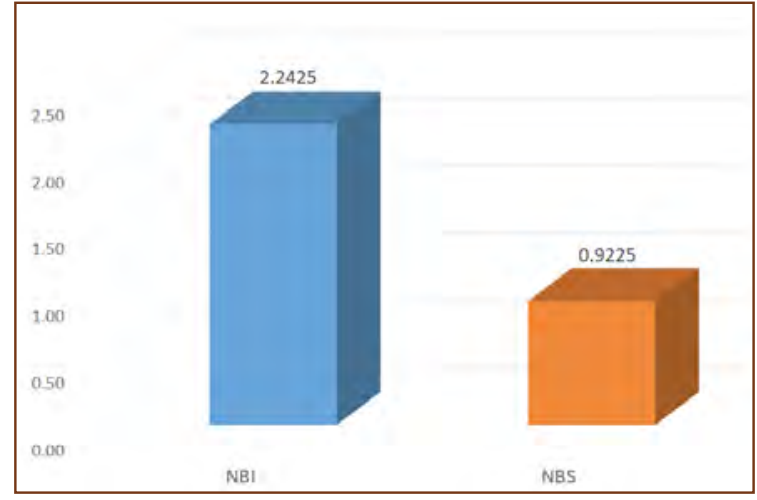

Figura 3. Mediana de la latencia en seguimiento del señalamiento para los grupos NBI y NBS.

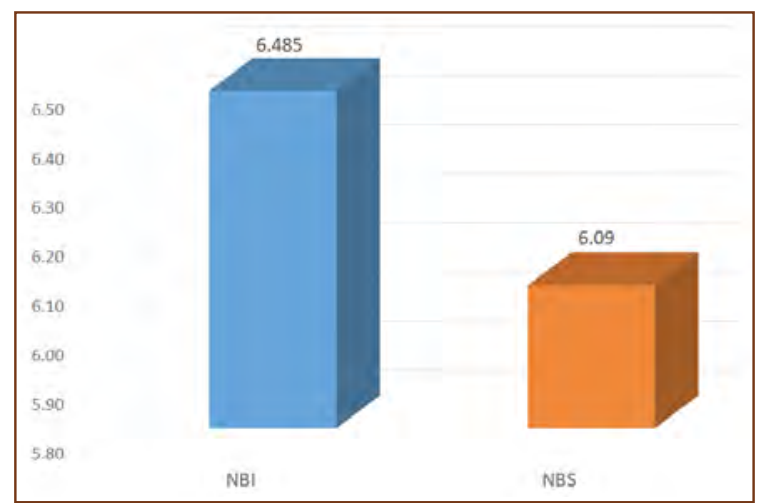

Figura 4. Mediana de la latencia en seguimiento de la mirada para los grupos NBI y NBS..

\section{DISCUSIÓN}

Este estudio es un acercamiento a pensar el rol de la vulnerabilidad social sobre el desarrollo de la comunicación temprana. El mismo tenía el objeto de estudiar la modulación que ejerce el nivel socioeconómico en las primeras habilidades comunicativas del infante. Se trabajó para cumplir con tal fin con el seguimiento de la mirada y el señalamiento, habilidades que se desarrollan durante los primeros años de vida. No se encontraron diferencias en el desempeño de estas habilidades en relación al nivel socioeconómico al que pertenecía el infante. Estos resultados aún no son concluyentes ya que hay aspectos a mejorar a nivel metodológico. Por un lado se debe aumentar la cantidad de participantes, por otro mejorar la homogeneidad de la muestra y por último realizar confiabilidad interobservadores.

A partir de los resultados se podría afirmar que en un entorno de interacción y con una base genética ya constituida, se esperaría que estas habilidades se desarrollen. Además, estas capacidades de la atención conjunta no implicarían capacidades de lenguaje oral, juego de ficción y habilidades mentalistas, en donde según Rivière ya es necesario el manejo consciente de recursos simbólicos y de control metacognitivo. Estas capacidades Rivière las agrupa en las funciones de tipo cuatro, adquiridas en la escolarización formal, y en las cuales ya se encuentran diferencias significativas en entornos con necesidades básicas satisfechas e insatisfechas (Farah \& Noble, 2004; Musso, 2010; Coronel, Lacunza, \& Gonzalez, 2006; Schady, 2011).

Además, estos resultados son consistentes con algunos de los estudios ya revisados, como el de Saxon y Reilly (1999), en donde no muestra correlaciones significativas entre el ESE y la atención conjunta entre madres y bebés. Si encontraron diferencias según sexo, habiendo mas interacciones con las madres que con los padres. Si bien estos trabajos no muestran correlaciones significativas entre el ESE y la atención conjunta entre madres y bebés, la importancia de replicar este tipo de investigaciones para ver si se encuentra algún tipo de relación entre estas variables reside en el hecho en que se ha reportado que estas habilidades son precursoras de otros procesos indispensables en el desarrollo del niño, como la teoría de la mente y el lenguaje (Kristen et al., 2011).

Los estudios ya mencionados de Tomasello y Todd (1983), Tomasello y Farrar (1986) también sugieren que lo que más influye en los episodios de atención conjunta son las estrategias y las conductas comunmente utilizadas por los cuidadores durante la misma, que afecta tanto a su ocurrencia, su cualidad y duración, como la capacidad didáctica del episodio de atención conjunta. Se podría afirmar que la calidad de las interacciones, el temperamento de los infantes y la escolaridad de los padres explican una mayor fracción de la variablidad de la atención conjunta que la riqueza de los hogares (Schady, 2011).

Se debe recordar que el constructo de vulnerabilidad social es multidimensional, es decir tiene en cuenta el nivel de escolaridad de los padres, el tipo de vivienda, entre otros, además del nivel económico. Sería interesante para próximas investigaciones agregar las variables de temperamento, estilos de crianza e iniciación de la atención conjunta, para obetener una mirada más abarcativa sobre el tema. También, realizar un estudio longitudinal que tenga en cuenta los trayectos de desarrollo de la atención conjunta.

Estos resultados podrían ser útiles como una primera aproximación al campo, para ser profundizados luego. La importancia de este tipo de estudios estriba en generar concientización en las políticas públicas y en la población de la importancia de la estimulacion temprana orientada hacia la comunicación preverbal. El infante precisa recibir estímulos a diario, desde el momento de su nacimiento. Si recibe estímulos pobres, de una forma irregular o en cantidad insuficiente, el cerebro no desarrolla adecuadamente sus capacidades al ritmo y con la calidad que cabría esperar. 
Sin embargo, a pesar de que el estudio de las competencias comunicacionales durante los primeros años de vida ha ido avanzando en los últimos años, aún se verifica una escasez de estudios empíricos que propongan abordar en forma simultánea la asociación entre competencias de comunicación, temperamento y su modulación por vulnerabilidad social (e.g., Hustedt \& Raver, 2002).

En tal sentido, el presente proyecto propone en un segundo momento una línea de trabajo elaborada para analizar tales aspectos, con el fin de contribuir no sólo a identificar los mecanismos subyacentes a tales asociaciones (interés básico), sino también a la generación de información pertinente para el diseño de intervenciones orientadas a optimizar las competencias de comunicación en fases tempranas del desarrollo (interés aplicado).

\section{REFERENCIAS BIBLIOGRÁFICAS}

American Psychological Association (1992). Ethical principles of psychologists and code of conduct. American Psychologist, 47, 1597-1611.

Baldwin, D. A. (1995). Understanding the link between joint attention and language. En C. Moore \& P.J. Dunham (Eds.). Joint attention: Its origins and role in development (pp. 131-158). Hillsdale, NJ: Lawrence Erlbaum Associates, Inc.

Billard, C., Bricout, L., Ducot, B., Richard, G., Ziegler, J., \& Fluss J. (2010). Evolution of competence in reading, spelling and comprehension levels in low socioeconomic environments and impact of cognitive and behavioral factors on outcome in two years. Revue Epidiemologique Sante Publique, 58, 101-110.

Bottem-Beutel, K. (2016). Associations between joint attention and language in autism spectrum disorder and typical development: A systematic review and meta-regression análisis. Autism Research, 9, $1021-1035$.

Bruner, J. (1995). From joint attention to the meeting of minds: An introduction. En C. Moore \& P. J. Dunham (Eds.), Joint Attention: Its Origin and Role in Development, pp. 189-203. Hillsdale, N. J.: Lawrence Erlbaum.

Brooks, R., \& Meltzoff, A. (2005). The development of gaze following and its relations to language. Developmental Science, 8, 535-543.

Colonnesi, G., Stams, I., Koster, I., \& Noom, M. (2010). The relation between pointing and language development: A meta-analysis. Developental Review, 4, 353-366

Carpendale, J. \& Lewis, C. (2004). Constructing an understanding of mind. The development of children's social understanding within social interaction. Behavioral and Brain Science, 27, 79151.

Carpenter, M., Nagell, K., \& Tomasello, M. (1998). Social cognition, joint attention, and communicative competence from 9 to 15 months of age. Monographs of the Society for Research in Child Development, 63, 1-174.

Charman, T. (2003). Why is joint attention a pivotal skill in autism. Philosophical Transactions of the Royal Society of London, 358, 315-324.

Coronel, P., Lacunza, A. \& González, N. (2006). Las Habilidades Cognitivas en niños privaods culturalmente. Resultados preliminares de la primera fase de evaluación. Ridep, 22, 49-74.

Dockrell, J.E., Stuart, M. \& King, D. (2010). Supporting early oral language skills for english language learners in inner city preschool provision. British Journal of Educational Psychology, 80, 497-516.

Escudero, A., Carranza, J. \& Huescar, E. (2013). Aparición y desarrollo de la atención conjunta en la infancia. Anales de Psicología, 29, 2-13.

Farah, M. \& Noble, K. (2004). Poverty, privilege, and brain development: empirical findings and ethical implications. Neuroethics, 9, 23-35.

Hustedt, J., \& Raver, C. (2002). Scaffolding in lowincome mother-child dyads: Relations with joint attention and dyadic reciprocity. International Journal of Behavioral Development, 26, 113-119.

INDEC, (2000). Hogares particulares con poblaciones objetivo: Perfil sociodemográfico. Documento de Trabajo $N^{\circ} 36$. Buenos Aires: Instituto Nacional de Estadística y Censo - Ministerio de Economía de la Nación.

Jenkins, C. (2003). Building better health. Washington: Panamerican Health Organization.

Kristen, S., Sodian, B., Thoermer, C., \& Perst, H. (2011). Infants' joint attention skills predict toddlers' emerging mental state language. Developmental Psychology, 47, 1207-1219.

Lipina, S.J., Martelli, M.I., Vuelta, B. \& Colombo, J.A. (2005). Performance on the A-not-B task of Argentinian infants from unsatisfied and satisfied basic needs homes. International Journal of Psychology, 39, 49-60.

McLaughlin, K.A., Breslau, J., Green, J.G., Lakoma, M.D., Sampson, N.A., Zaslavsky, A.M., Kessler, R.C. (2011). Childhood socio-economic status and the onset, persistence, and severity of DSM-IV mental disorders in a US national sample. Social Science and Medicine, 73, 1088-1096.

McGillion, M., Herbert, J., Pine, J. M., KerenPortnoy, T.,Vihman, M., \& Matthews, D. (2013). Supporting early vocabulary development: What sort of responsiveness matters? Transactions on Autonomous Mental Development, 5, 240-248. doi:10.1109/TAMD.2013.2275949

McGillion, M., Herbert, S., Pine, J., Vihman, M., dePaolis, R., Karen-Portnoy, T., Matthews, D. (2016). What Paves the Way to Conventional Language? 
The Predictive Value of Babble, Pointing, and Socioeconomic Status. Chile Developmnet, 10, 1-11.

Morales, M., Mundy, P., Delgado, C., Yale, M., Messinger, D., Neal, R., \& Schwartz, H. (2000). Responding to joint attention across the 6 - through 24 -month age period and early language acquisition. Journal of Applied Developmental Psychology; 21, 283-298.

Mundy, P. \& Jarrold, W. (2010). Infant Joint Attention, Neural Networks and Social Cognition. Neural Networks, 23, 985-997.

Murza, K. \& Schwartz, J. (2016). Joint attention interventions for children with autism spectrum disorder: a systematic review and metaanalysis. International Journal of Language \& Communication Disorders, 3, 236-251.

Musso, M. (2010). Funciones ejecutivas: Un estudio de los efectos de la pobreza sobre el desempeño ejecutivo. Interdisciplinaria, 27.

Rivière, A. (1999). Desarrollo y educación: El papel de la educación en el diseño del desarrollo humano. En M. Belinchón, A. Rosa, M. Sotillo e I. Marichalar (Eds.), Ángel Rivière. Obras escogidas, Vol III (pp.203-242). Madrid: Panamericana.

Saxon, T., \& Reilly, J.T. (1999). Joint attention and toddler characteristics: Race, sex and socioeconomic status. Early Child Development and Care, 149, 59-69.

Schady, N. (2011). Parents' education, mothers' vocabulary, and cognitive development in early childhood: longitudinal evidence from Ecuador. American Journal of Public Health, 101, 2299 2307.

Sheinkopf, S.J., Mundy, P., Claussen, A.H., \& Willoughby, J. (2004). Infant joint attention skill and preschool behavioral outcomes in at-risk children. Development and Psychopathology, 16, 273-291.
Sigman, M., \& Kasari, C. (1995). Joint attention across contexts in normal and autistic children. En C. Moore \& P. J. Dunham (Eds.), Joint Attention: Its Origin and Role in Development, 189-203. Hillsdale, N.J.: Lawrence Erlbaum.

Stern, D. (1985). The interpersonal world of the infant. Nueva York: Basic Books.

Stevenson, M.B., Roach, M.A., Leavitt, L.A., Miller, J.F., \& Chapman, R.S. (1998). Early receptive and productive language skills in pre-term and fullterm 8-month-old infants. Journal of Psycholinguist Research, 17, 169-183.

Tomasello, M. \& Farrar, M.J. (1986). Joint attention and early language. Child Development, 57, 14541463.

Tomasello, M. (1995). Joint attention as social cognition. En C. Moore \& P. J. Dunham (Eds.), Joint Attention: Its Origin and Role in Development, pp. 103-130. Hillsdale, N.J.: Lawrence Erlbaum.

Tomasello, M., \& Carpenter, M. (2005). The emergence of social cognition in three young chimpanzees. Monographs of the Society for Research in Child Development, 70, (1, Serial No. 279).

Tomasello, M., \& Todd, J. (1983). Joint attention and lexical acquisition style. First Language, 4, 197 212.

Trevarthen, C. (1990). Signs before speech. In T. A. Sebeok \& J. Umiker-Sebeok (Eds.), The semiotic web, 689- 755 .

Ulvund, S. E., \& Smith, L. (1996). The predictive validity of nonverbal communicative skills in infants with perinatal hazards. Infant Behavior and Development, 19, 441-449.

UNICEF (2005). Estado Mundial de la Infancia. Washington DC: Fondo de las Naciones Unidas para la infancia. Naciones Unidas. 